\title{
ESTUDIO TEÓRICO DE LAS PROPIEDADES ELECTRÓNICAS EN 4-X-1,2-BENZOQUINONAS SUSTITUIDAS
}

\author{
Ronald Ruíz ${ }^{1}$, Hélmer Lezama ${ }^{1}$, Rodolfo Pumachagua ${ }^{1 *}$
}

\begin{abstract}
RESUMEN
En este trabajo se analiza descriptores globales como la dureza, electronegatividad, nucleofilia y potencial químico, e índices de reactividad local. Utilizamos la Teoría del Funcional de Densidad (DFT) a un nivel de cálculo B3LYP/6-31G. Con los índices de reactividad local se analiza el carácter nucleófilo/electrófilo por efecto de grupos sustituyentes sobre el sistema 1,2-benzoquinona. Con los descriptores globales de reactividad, se obtiene relaciones de correlación aceptables así como el efecto de los grupos sustituyentes sobre el espectro Uvvis.
\end{abstract}

Palabras claves: Índice de Fukui, benzoquinona, descriptores globales, espectro Uv-visible.

\section{THEORETICAL STUDY TO THE ELECTRONIC PROPERTIES IN 4-X-1,2-BENZOQUINONES SUBSTITUTED}

\begin{abstract}
This paper discusses global descriptors such as hardness, electronegativity, nucleofilia and chemical potential and local reactivity indexes. We use the method of the density functional (DFT) to a level of calculation B3LYP/6-31G. With local reactivity indexes analyzed the nucleophile/electrophile character by the effect of substituents on the system 1,2-benzoquinone. With global descriptors of reactivity, we obtained acceptable correlations as well as the effect of substituents on the Uv-vis spectrum groups.
\end{abstract}

Key words: Fukui index, benzoquinone, global descriptors, spectra Uv-visible.

\section{INTRODUCCIÓN}

Las quinonas han sido conocidas desde la antigüedad por sus propiedades tintóreas.

Se conoce una diversidad de moléculas con núcleo quinona, alrededor de unos 200 aislados de la naturaleza 1, que tienen múltiples funciones; sin embargo, pocas han sido las investigaciones

\footnotetext{
${ }^{1}$ Universidad Nacional Federico Villarreal, Facultad de Ciencias Naturales y Matemática, Laboratorio de Química Teórica, Jr. Chepén s/n (El Agustino) Perú. rpumachagua@gmail.com
} 
conducentes al establecimiento de un inventario nacional de los productos naturales propios amparados con estudios experimentales, teórico-experimentales o puramente teóricos.

El objetivo de la química cuántica es resolver los problemas de la química, donde predice propiedades que son difíciles de obtener por medios experimentales, como la reactividad, estados de transición, propiedades ópticas entre otras. Uno de los métodos más utilizados para calcular las propiedades electrónicas es la Teoría del Funcional de Densidad (DFT).

\section{FUNDAMENTO TEÓRICO}

El primer teorema de Hohenberg-Khon ${ }^{2}$ afirma que la densidad electrónica, $\rho(r)$, se determina por el potencial externo del núcleo, $v(r)$. La normalización de la densidad electrónica determina el número total de electrones, $\mathrm{N}$ :

$$
\int \rho(r) d r=\mathrm{N}
$$

$\mathrm{N}$ y $v(r)$ determinan el Hamiltoniano molecular, $H_{o p}$ dentro de la aproximación de BornOppenheimer, despreciando los efectos relativistas. Conceptos químicos como la electronegatividad y dureza, fueron desarrolladas dentro del DFT Conceptual propuestos por Parr y colaboradores ${ }^{3}$. La electronegatividad $(x)$, es el negativo del potencial químico, definido por un sistema de $\mathrm{N}$ electrones con energía total E y potencial externo $v(\bar{r})$.

$$
x=-\mu=-\left(\frac{\partial E}{\partial N}\right)_{v(\bar{r})}
$$

Observamos que el potencial químico $(\mu)$, describe los cambios en la energía electrónica con respecto al número de electrones que está asociado con la capacidad de transferencia de carga del sistema en su estado fundamental.

La dureza $(\eta)$, está definida como la segunda derivada correspondiente, y se muestra a continuación:

$$
\eta=\left(\frac{\partial^{2} E}{\partial N^{2}}\right)_{v(\bar{r})}=\left(\frac{\partial \mu}{\partial N}\right)_{v(\bar{r})}
$$

La blandura ( $\mathrm{S}$ ) es el reciproco de la dureza; $\mathrm{S}=1 / \eta$.

Una función de onda de $\mathrm{N}$ partículas necesita solo de $\mathrm{N}$ y $v(\bar{r})$ para su descripción total. Mientras x y $\eta$ miden la respuesta del sistema cuando $\mathrm{N}$ varía, siendo $v(\bar{r})$ constante. El comportamiento del sistema por un cambio en $v(\bar{r})$ con $\mathrm{N}$ fijo, es dado por la función de respuesta de densidad lineal; esto se logra con funciones basadas en la densidad electrónica como en el DFT. La respuesta lineal de la nube electrónica de un sistema por efecto de un campo eléctrico externo débil es medida en términos de la polarizabilidad del dipolo eléctrico estático.

Si $\in_{\text {номо }}$ y la $\in_{\text {LUMO }}$ son las energías de los orbitales del más alto ocupado y el más bajo desocupado respectivamente, tanto el HOMO como el LUMO son denominados orbitales frontera que intervienen en la reactividad de sistemas moleculares; entonces se escribe una ecuación usando el teorema de Koopman ${ }^{4}$ : 


$$
\begin{aligned}
& x=-\frac{\epsilon_{\text {HOMO }}+\epsilon_{\text {LUMO }}}{2} \approx-\mu \\
& \eta=\epsilon_{L U M O}-\epsilon_{\text {Hомо }}
\end{aligned}
$$

Estas también se relacionan con la energía de ionización y la afinidad electrónica, respectivamente. El índice de nucleofilia, $(\mathrm{N})$, para un sistema dado, se define entonces como:

$$
N=\epsilon_{\text {Hомо }(\mathrm{Nu})}-\epsilon_{\text {Hомо }}(\mathrm{TCE})
$$

donde, $\in_{\text {номо }}(\mathrm{Nu})$ es la energía del HOMO del nucleófilo, y $\in_{\text {номо(тCE) }}$ corresponde a la energía del HOMO del tetracianoetileno (TCE) tomado como el valor de referencia ${ }^{5}$.

Respecto al índice de electrofilia $(\omega)$, considerado una medida del poder electrofilico ${ }^{3}$, tenemos:

$$
\omega=\frac{\mu^{2}}{2 \eta}=\frac{\chi^{2}}{2 \eta}
$$

La función de Fukui es el más importante índice local de reactividad. Se define como:

$$
f(\vec{r})=\left(\frac{\partial \rho(\vec{r})}{\partial N}\right)_{v(\vec{r})}=\left(\frac{\delta \mu}{\delta v(\vec{r})}\right)_{N}
$$

Debido a las discontinuidad en la pendiente de la curva de $\rho(\vec{r})$ versus $\mathrm{N}$, tres funciones de Fukui $\left(f_{\kappa}^{\alpha}, \alpha=+,-, 0\right)$ pueden ser reemplazados por las densidades electrónicas que se asocian, por la población electrónica respectiva $\left(q_{k}\right)$ como cargas de Mulliken ${ }^{6,7}$ :

a. Para un ataque nucleofílico:

$$
f_{\kappa}^{+}=q_{\kappa}(N+1)-q_{\kappa}(N)
$$

b. Para un ataque electrofílico:

$$
f_{\kappa}^{-}=q_{\kappa}(N)-q_{\kappa}(N-1)
$$

Para la determinación de propiedades como la polarizabilidad y energías de excitación, solo se requiere el conocimiento del resultado de la densidad de respuesta lineal ${ }^{8}$ en donde se da la propiedad de un cambio en el potencial externo cuando está fijo el número de electrones. En la década de los cuarenta los químicos R. B. Woodward y L. F. Fieser desarrollaron una relación en las transiciones electrónicas en dienos conjugados, para estas reglas se usan ciertos términos en las transiciones electrónicas ${ }^{9}$.

\section{ASPECTOS COMPUTACIONALES}

Los cálculos fueron obtenidos usando el programa GAUSSIAN09. Con respecto a los índices de Fukui, las estructuras se optimizaron empleando las funciones base: 6-31G mediante el funcional B3LYP. Todas las frecuencias vibracionales del oscilador armónico de las respectivas estructuras resultaron positivas, confirmando los mínimos de energía de la estructura optimizada. 


\section{RESULTADOS Y DISCUSIÓN}

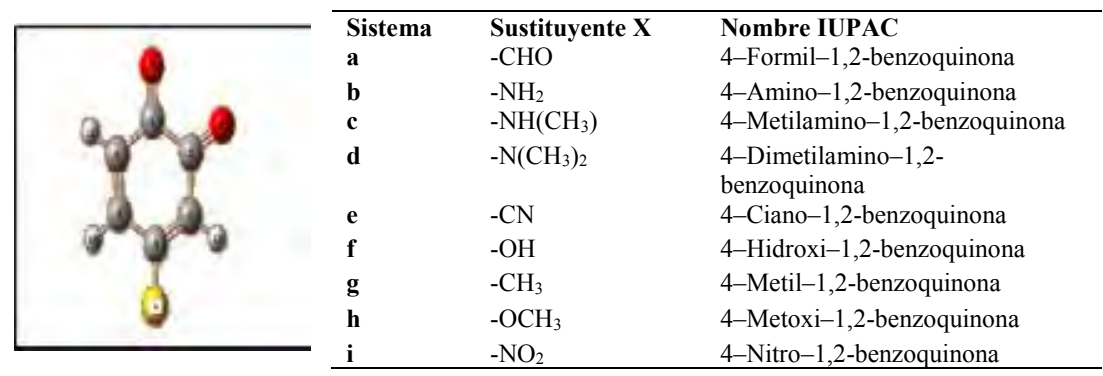

Figura 1. Sistemas de derivados: $4-\mathrm{X}-1,2$ benzoquinona.

\section{Índices de reactividad local en derivados de o-benzoquinona.}

Tabla 1. Índices de Fukui electrofílico en derivados de o-benzoquinona. B3LYP/6-31G.

\begin{tabular}{|c|c|c|c|c|c|c|c|c|c|c|}
\hline Sistema & $a$ & b & c & $d$ & e & $f$ & g & $\mathrm{h}$ & $i$ & $\mathrm{Bz}$ \\
\hline C1 & 0,031647 & 0,014338 & 0,013848 & 0,012305 & 0,036774 & 0,016005 & 0,038047 & 0,013042 & 0,033805 & 0,035507 \\
\hline $\mathrm{C} 2$ & 0,033635 & 0,008955 & 0,007933 & 0,008735 & 0,029811 & 0,010200 & 0,027818 & 0,009340 & 0,034455 & 0,035506 \\
\hline C3 & 0,048623 & 0,123142 & 0,123697 & 0,115945 & 0,046580 & 0,124130 & 0,044496 & 0,113735 & 0,047525 & 0,039928 \\
\hline C4 & $-0,000462$ & 0,006510 & 0,004905 & 0,004447 & 0,024932 & 0,027797 & 0,007727 & 0,036065 & 0,019121 & 0,034192 \\
\hline C5 & 0,041252 & 0,023651 & 0,020542 & 0,015840 & 0,045125 & 0,034010 & 0,038137 & 0,021257 & 0,047370 & 0,034193 \\
\hline C6 & 0,030698 & 0,047779 & 0,041345 & 0,039779 & 0,036088 & 0,067690 & 0,036969 & 0,061204 & 0,034257 & 0,039927 \\
\hline 07 & 0,172427 & 0,102290 & 0,092624 & 0,087280 & 0,195986 & 0,116142 & 0,185110 & 0,108212 & 0,195875 & 0,205741 \\
\hline 08 & 0,163434 & 0,158177 & 0,153205 & 0,144528 & 0,195574 & 0,149697 & 0,212411 & 0,142654 & 0,174264 & 0,205746 \\
\hline
\end{tabular}

Tabla 2. Índices de Fukui nucleofilico en derivados de o-benzoquinona. B3LYP/6-31G.

\begin{tabular}{|c|c|c|c|c|c|c|c|c|c|c|}
\hline Sistema & $a$ & b & c & d & e & $f$ & g & $\mathrm{h}$ & i & $\mathrm{Bz}$ \\
\hline C1 & 0,035920 & 0,059276 & 0,058642 & 0,055886 & 0,041412 & 0,057213 & 0,053454 & 0,052652 & 0,033614 & 0,050718 \\
\hline C2 & 0,050700 & 0,032090 & 0,033618 & 0,033752 & 0,048552 & 0,041499 & 0,048292 & 0,038735 & 0,044559 & 0,050718 \\
\hline C3 & 0,056030 & 0,050112 & 0,046586 & 0,041452 & 0,056885 & 0,051360 & 0,047954 & 0,048744 & 0,065863 & 0,045064 \\
\hline C4 & 0,004012 & 0,006756 & 0,011171 & 0,015839 & 0,031089 & 0,031763 & $-0,000568$ & 0,029961 & 0,026666 & 0,02737 \\
\hline C5 & 0,030577 & 0,050899 & 0,050705 & 0,052640 & 0,030598 & 0,045124 & 0,038647 & 0,052636 & 0,027624 & 0,02737 \\
\hline C6 & 0,038935 & 0,041994 & 0,040041 & 0,041107 & 0,042867 & 0,040301 & 0,041300 & 0,045333 & 0,040169 & 0,045064 \\
\hline 07 & 0,142744 & 0,161025 & 0,157590 & 0,154063 & 0,148000 & 0,162834 & 0,159040 & 0,159665 & 0,136701 & 0,162025 \\
\hline 08 & 0,149740 & 0,141765 & 0,136289 & 0,133674 & 0,151159 & 0,151973 & 0,156624 & 0,148057 & 0,144393 & 0,162025 \\
\hline
\end{tabular}
(a)
(b)
(c)
(d)

Figura 2. Regiones de densidad electrónica para la molécula "d": (a) Homo y (b) Lumo. Regiones de densidad electrónica para la molécula “i”: (c) Homo y (d) Lumo.

Las moléculas estudiadas están representadas en la figura 1. La tabla 1 muestra los valores de la función de Fukui para un ataque electrofílico $\left(f_{\kappa}^{-}\right)$. Los sustituyentes: $-\mathrm{NH}_{2},-\mathrm{NH}\left(\mathrm{CH}_{3}\right)$, $-\mathrm{N}\left(\mathrm{CH}_{3}\right)_{2},-\mathrm{OH},-\mathrm{CH}_{3}$ y $-\mathrm{OCH}_{3}$ son grupos dadores, que convierten al $\mathrm{O} 8$ como el sitio de mayor densidad electrónica, por la deslocalización electrónica en la nube $\pi$ generada por el sustituyente ${ }^{10,11}$. Para los sistemas con sustituyentes atractores: $-\mathrm{CHO},-\mathrm{CN}$ y $-\mathrm{NO}_{2}$, el O7 tiene la mayor densidad electrónica por efecto mesomérico, debido a la absorción de 
densidad electrónica del sustituyente desde la nube $\pi$ del anillo, específicamente de los carbonos: $\mathrm{C} 3$ y $\mathrm{C} 4$.

Cabe notar que la diferencia de valores del índice entre 07 y 08 es despreciable en un sistema sin grupo sustituyente $(\mathrm{B} z)$. Sin embargo, el efecto de un sustituyente atractor genera una diferencia mínima entre estos oxígenos, mientras que con grupos dadores es mayor.

Por otro lado, se observa al C3 como el sitio de mayor densidad electrónica en el anillo tanto en sistemas con sustituyentes dadores como atractores, debido a que este carbono se encuentra en la ruta de deslocalización electrónica, adquiriendo una carga negativa parcial. Los valores de la función de Fukui del C3 son mayores en sistemas con sustituyentes dadores que con atractores.

La tabla 2 muestra los valores de la función de Fukui para un ataque nucleofilico $\left(f_{\kappa}^{+}\right)$. Observamos que el $\mathrm{O} 7$ y $\mathrm{O} 8$ es el sitio de menor densidad electrónica, cuando se tiene en el sistema un sustituyente dador y atractor, respectivamente. Además, el sitio electrófilo es el $\mathrm{C} 3$ cuando tiene un sustituyente atractor, mientras que el $\mathrm{C} 1$ corresponde cuando tiene un sustituyente dador.

La figura 2 muestra regiones de densidad electrónica de los orbitales HOMO y LUMO para los sistemas "d" e "ij", con sustituyente dador y atractor, respectivamente. La figura 2(a) muestra el HOMO del sistema "d" para un ataque electrofílico, se presenta la región de mayor densidad electrónica en los carbonos: C2, C3 y C4, así como en los átomos de oxígeno. En la figura 2(b) se muestra el orbital LUMO para un ataque nucleofílico, los carbonos: C1, C2, C3 y C6 aceptan electrones. En la figura 2(c) se observa el orbital HOMO del sistema "i' para un ataque electrofílico, la mayor densidad electrónica se encuentra entre los átomos de oxígeno en contraste con la figura 2(d) que corresponde al orbital LUMO del sistema "ii" para un ataque nucleofílico.

\section{Estudio de la reactividad global en derivados de o-benzoquinona.}

Tabla 3. Índices globales de reactividad en derivados de o-Bz. B3LYP/6-31G

\begin{tabular}{ccccccc}
\hline Sistema & $\begin{array}{c}\text { HOMO } \\
(\mathrm{ev})\end{array}$ & $\begin{array}{c}\text { LUMO } \\
(\mathrm{ev})\end{array}$ & $n(\mathrm{ev})$. & $x$ & $\omega(\mathrm{ev})$. & $N(\mathrm{ev})$. \\
$\mathbf{a}$ & $-7,3822$ & $-4,4458$ & 2,9364 & 0,2173 & 0,0693 & 1,7364 \\
$\mathbf{b}$ & $-6,2894$ & $-3,2542$ & 3,0352 & 0,1754 & 0,0467 & 2,8292 \\
$\mathbf{c}$ & $-6,1522$ & $-3,1432$ & 3,0090 & 0,1708 & 0,0439 & 2,9663 \\
$\mathbf{d}$ & $-5,9446$ & $-3,1372$ & 2,8074 & 0,1669 & 0,0391 & 3,1739 \\
$\mathbf{e}$ & $-7,5906$ & $-4,5729$ & 3,0177 & 0,2235 & 0,0754 & 1,5279 \\
$\mathbf{f}$ & $-6,9112$ & $-3,6863$ & 3,2248 & 0,1947 & 0,0611 & 2,2074 \\
$\mathbf{g}$ & $-6,8603$ & $-3,6877$ & 3,1726 & 0,1938 & 0,0596 & 2,2583 \\
$\mathbf{h}$ & $-6,7446$ & $-3,6654$ & 3,0792 & 0,1913 & 0,0563 & 2,3739 \\
$\mathbf{i}$ & $-7,7667$ & $-4,8869$ & 2,8798 & 0,2325 & 0,0778 & 1,3519 \\
$\mathbf{B z}$ & $-7,0148$ & $-3,8774$ & 3,1375 & 0,2001 & 0,0628 & 2,1037 \\
\hline
\end{tabular}




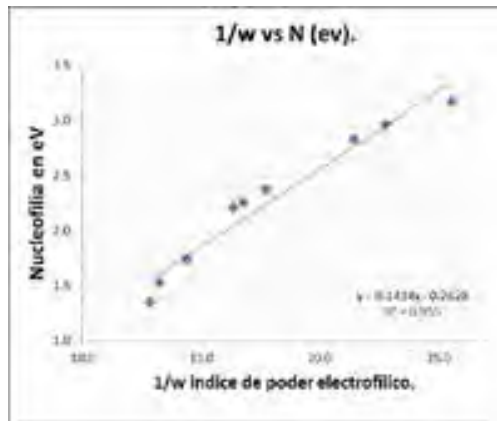

Figura 3. Índice de poder electrofílico vs. nucleofilia.

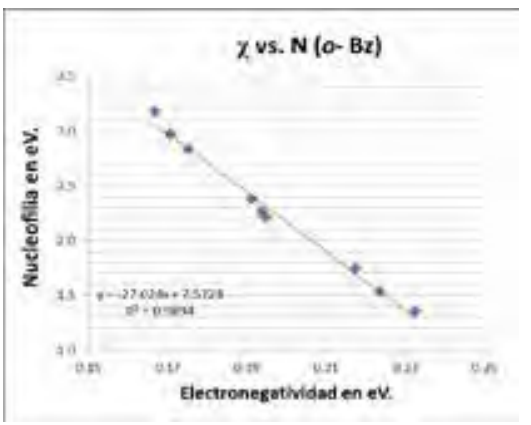

Figura 4. Electronegatividad vs. nucleofilia.

En la tabla 3 se tiene los descriptores globales de reactividad con sustituyentes dadores: "b", "c", “d", "f", "g" y "h" y sustituyentes atractores: "a", "e" e "i”". La dureza, $\eta$, determina la reactividad del sistema, observamos que se ordena así: "f">"g">"h">"b">"c">>d". En los sistemas: "b", “c”" $\mathbf{y}$ "d" la dureza disminuye por la sustitución de grupos metilos por hidrógenos, este factor influye en mayor grado que la naturaleza del sustituyente ${ }^{12}$.

El grupo - $\mathrm{OH}$ incrementa la dureza del sistema, mientras más electronegativo es el átomo en el sustituyente dador, más estable es el sistema, aun sobre el par de electrones libres del nitrógeno en sistemas con sustituyente amino. Este comportamiento por grupos sustituyentes fue observado en otros estudios sobre la dureza en compuesto aromáticos ${ }^{13}$. El sistema "d" tiene el valor más bajo de dureza, disminuyendo más el "band gap” de energía.

La nucleofilia $(N)$ es la inversa del índice de electrofilia ${ }^{14}$, tiene una relación proporcional con la nucleofilia teórica. En la figura 3 se observa que en todos los sistemas el valor de la nucleofilia y la inversa del índice de electrofilia, $\omega$, muestra una proporcionalidad entre los valores. Mayr y colaboradores ${ }^{15}$ propusieron escalas de electrofilia/nucleofilia en reacciones químicas de índoles con diversos tipos de sustituyentes, obteniendo dependencia lineal semejante con los índices de reactividad teórico realizado en este trabajo.

Una molécula con alto carácter nucleófilo tiene el potencial químico $(\mu)$ más alto, que corresponde al valor más bajo de electronegatividad $(x)$. Los valores de la tabla 3 muestran que el más bajo en electronegatividad tiene el valor más alto en nucleofilia. En la figura $4 \mathrm{se}$ nota la relación inversamente proporcional entre la nucleofilia y la electronegatividad.

La tabla 4 tiene valores de transición electrónica para los sistemas $o$-benzoquinona sustituidas. Los sistemas con sustituyente atractor experimentan transiciones $n \rightarrow \pi^{*}$ y poseen menor energía de transición y mayor longitud de onda. El efecto contrario se obtiene en sistemas con sustituyente dador donde ocurre una transición $\pi \rightarrow \pi^{*}$.

Las figuras: 5, 6 y 7 permiten analizar el aumento y la disminución de la energía de transición por efecto del grupo sustituyente respecto al sistema $o$-benzoquinona sin sustituir. La molécula $o$-benzoquinona presenta dos bandas de absorción donde la más intensa está próxima a 400 
$\mathrm{nm}^{16}$. El sistema atractor " $\mathbf{i}$ " presenta solo una banda intensa en el barrido, similar a los sistemas: "a" y "e", con una longitud de onda cercana a $400 \mathrm{~nm}$. En el sistema dador "g" la banda cercana a $400 \mathrm{~nm}$ disminuye en intensidad respecto a o-Bz, similar a los sistemas: "b", "c" y "d".

\section{Estudio del análisis espectroscópico en derivados de o-benzoquinona.}

Tabla 4. Estados excitados en derivados de $o$-benzoquinona. TDDFT B3LYP/6-31G.

\begin{tabular}{ccccccc}
\hline Sistema & Oscilador & $\begin{array}{c}\text { Estado } \\
\text { excitado }\end{array}$ & $\begin{array}{c}\text { Orbital } \\
\text { ocupado }\end{array}$ & $\begin{array}{c}\text { Orbital } \\
\text { vacío }\end{array}$ & $\mathrm{E}(\mathrm{ev})$ & $\lambda(\mathrm{nm})$ \\
$\mathbf{a}$ & 0,0399 & 4 & 34 & 36 & 3,0149 & 411,23 \\
$\mathbf{b}$ & 0,1237 & 4 & 29 & 33 & 4,7292 & 262,17 \\
$\mathbf{c}$ & 0,1703 & 4 & 33 & 37 & 4,5465 & 272,70 \\
$\mathbf{d}$ & 0,1762 & 4 & 37 & 41 & 4,3192 & 287,06 \\
$\mathbf{e}$ & 0,0954 & 4 & 31 & 35 & 4,5836 & 270,49 \\
$\mathbf{f}$ & 0,1137 & 4 & 29 & 33 & 4,8526 & 255,50 \\
$\mathbf{g}$ & 0,0743 & 4 & 29 & 33 & 5,0730 & 244,40 \\
$\mathbf{h}$ & 0,1531 & 4 & 33 & 37 & 4,6725 & 265,35 \\
$\mathbf{i}$ & 0,0330 & 4 & 38 & 40 & 2,9862 & 415,19 \\
$\mathbf{B z}$ & 0,0490 & 3 & 27 & 29 & 3,0844 & 401,97 \\
\hline
\end{tabular}

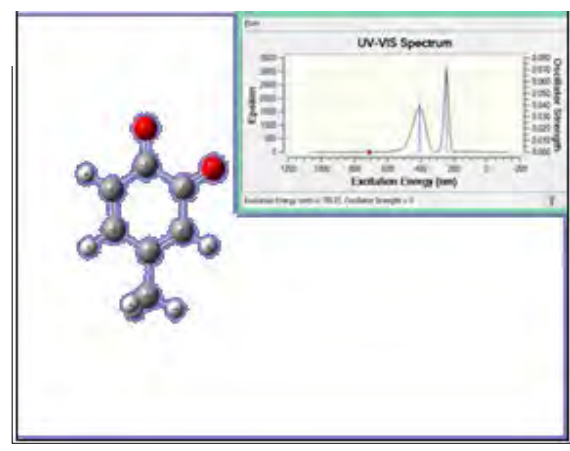

Figura 5. Espectro UV-visible del sistema "g"

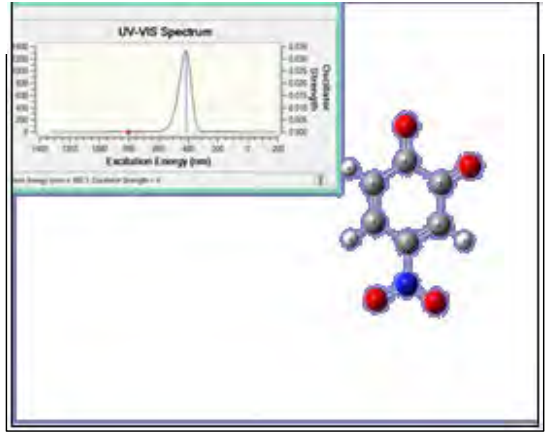

Figura 6. Espectro UV-visible del sistema "ii".

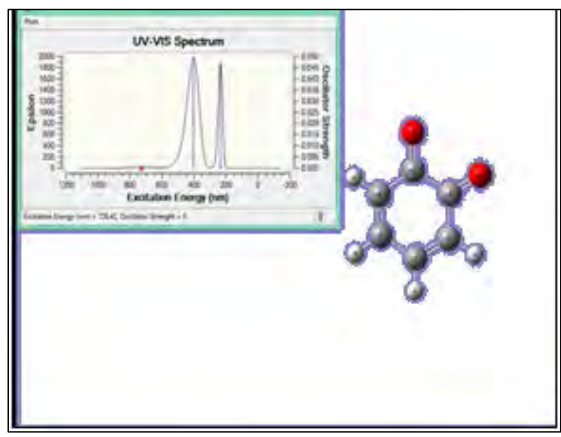

Figura 7. Espectro UV-visible del sistema $o$-Bz. 


\section{CONCLUSIONES}

Los cálculos realizados con la Teoría del Funcional de Densidad (DFT), a un nivel B3LYP/6$31 \mathrm{G}$ describen satisfactoriamente las propiedades de reactividad en $o$-benzoquinonas monosustituidas. Los valores de los índices de Fukui, para un ataque electrofílico y/o nucleofilico, cuando se tiene variados sustituyentes: dador y atractor, concuerdan con los resultados experimentales. Además, la Teoría del Funcional de Densidad dependiente del Tiempo (TDFT), es una buena herramienta en la discusión de las transiciones electrónicas cuando se analiza el efecto de grupos sustituyentes sobre el espectro Uv-visible.

\section{AGRADECIMIENTO}

Los autores agradecen al Dr. William Tiznado Vásquez de la Universidad Andrés Bello, Santiago de Chile, por brindarnos soporte computacional.

\section{REFERENCIAS BIBLIOGRÁFICAS}

1. R.H. Thomson, "Naturally Occurring Quinones". Segunda edición. Academic Press Inc. (London) LTD. 1971, 93-197.

2. Hohenberg, P. \& Kohn, W. Inhomogeneous Electron Gas. Phys. Rev. B, 1964; 136: 864871.

3. Parr, R.G. \& Yang, W., Density-functional theory of atoms and molecules. New York: Oxford University Press. 1989.

4. Koopmans, T.A., About the allocation of wave functions and own values to the single electrons of an atom. Physical, 1933; 1: 104-113.

5. Patricia Pérez, Luis Domingo, Mario Duque-Noreña \& Eduardo Chamorro. A condensedto-atom nucleophicility index. An application to the director effects on the electrophilic aromatic substitutions. Journal of Molecular Structure: THEOCHEM, 2008; 895: 8691.

6. Mulliken, R. S., A new electroaffinity scale; together with data on valence states on valence ionization potentials and electron affinity. J. Chem. Phys, 1934; 2: 782-793.

7. Pratim Kumar Chattaraj, Utpal Sarkar, Debesh Ranjan Roy (2005). Electrophilicity Index. Chem. Rev, 2006; 106: 2065-2091.

8. Runge, Erich \& Gross, E. K. U., Density-Functional Theory for Time-Dependent Systems. Physical Review Letters, 1984; 52: 997-1000.

9. Woodward, Robert Burns, Structure and the Absorption Spectra of $\alpha, \beta$-Unsaturated Ketones. J. Am. Chem. Soc, 1941; 63: 1123-1126.

10. H. Szatyzowicz, T. M. Krygowski, M. Palusiak, J. Poater \& M. Solá. Routes of $\pi$-electron delocalization in 4-substituded-1,2-benzoquinone. J. Org. Chem., 2011; 76: 550-556.

11. John Vinokur, Reactions of Substituted Quinones. Department of Chemistry. McGill University. Montreal Canada. 1972. 
12. Chang-Guo Zhan, Jeffrey A. Nichols \& David A. Dixon. Ionization potential, electron affinity, electronegativity, hardness, and electron excitation energy: Molecular properties from density functional theory orbital energies. J. Phys. Chem. A, 2003; 107: 41844195.

13. Nancy Montes, Angelina Hormaza. Comparación de los Índices Locales de Reactividad Fukui de una Serie de Aldehídos. Rev Soc Quím Perú, 2008; 74: 247-251.

14. José L. Gásquez, Andrés Cedillo \& Alberto Vela. Electrodonating and electroaccepting powers. J. Phys. Chem. A, 2007; 111: 1966-1970.

15. S. Lakhdar, M. Westermaier, F. Terrier, R. Goumont, T. Boubaker, A. R. Ofial \& H. Mayr, Nucleophilic reactivities of indoles. J. Org. Chem., 2006; 71: 9088-9095.

16. Jerome W. Sidman, The electronic spectra and structure of the quinones. J. Am. Chem. Soc., 1956; 78: 4567-4572. 